\title{
A Novel Nasal Mask Assembly Provided CPAP Pre-Oxygenation, Positive Pressure Ventilation and Apnoeic Oxygenation during General Anaesthesia Induction in an Obese Patient with Suspected OSA, Difficult Airway and Poor Face-Mask Fit during Bronchoscopy
}

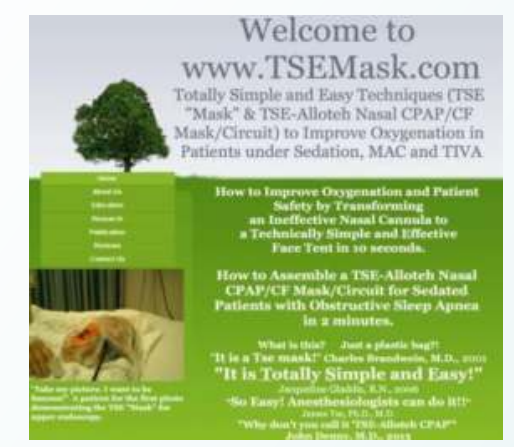

\author{
Nicole Grayer, MD, Vanessa Rodriguez, CRNA, APN, MS, \\ Ryan Sison, MD and James Tse, PhD, MD \\ Dept. of Anesthesiology, Rutgers Robert Wood Johnson Medical School, \\ New Brunswick, New Jersey, USA
}

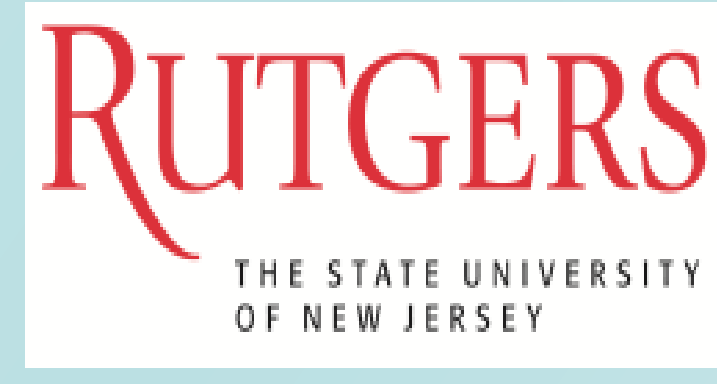

ePoster Presentation (13AP01-5) at the Euroanaesthesia 2016, London, UK, 28 -30 May, 2016

Background: Obese patients often present difficulties with face mask ventilation during general anaesthesia (GA) induction.

A simple nasal TSE-PAP mask assembly has been shown to maintain oxygenation in sedated obese OSA patients by providing CPAP or BiPAP and to improve ventilation in anaesthetized patients with poor face-mask fit(Photo 1). ${ }^{1-3}$

We presented its use in an obese patient with a difficult airway..

Case Report: A 65 y/o obese (BMI $34 \mathrm{~kg} / \mathrm{m}^{2}$ ) female with metastatic lung cancer to the brain, $\mathrm{s} / \mathrm{p}$ chemotherapy and radiation therapy presented for bronchoscopy to evaluate hemoptysis. CTA revealed a large right hilar mass (8.5 $\mathrm{cm}$ ) encased pulmonary artery and mainstem bronchus.

She had a full round face, a Class III airway, poor dentition, a large tongue and suspected OSA.

The patient gave consent to use a nasal mask for GA induction and for photography/case report.

An infant mask (size \#2) with a fully inflated air cushion was secured over her nose with head straps and connected to a breathing circuit with $6 \mathrm{~L} / \mathrm{min}_{2}$ and 5-6 $\mathrm{cm} \mathrm{H}_{2} \mathrm{O}$ CPAP delivered through the anaesthesia machine(Photo 1).

Her $\mathrm{O}_{2}$ saturation (Sat) increased to $100 \%$ from $93 \%$. GA was induced with fentanyl and propofol. Her mouth was closed and a tight nose-mask seal was obtained with one hand and positive pressure ventilation was easy with the other hand(Photo 2).

Succinylcholine was then given. With the nasal mask assembly providing apneic oxygenation, endotracheal intubation (ETI) was performed using a video-

laryngoscope (VL). Her $\mathrm{O}_{2}$ Sat was $100 \%$ throughout ETI.

Bronchoscopy revealed active arterial bleeding in the right main bronchus requiring urgent endovascular

embolization. She was placed in the right lateral decubitus position. Left radial arterial catheter was inserted to monitor blood pressure.

The patient was stabilized and transported to the Interventional Radiology Suite for bronchial artery embolization. She tolerated the procedure well without any complications and bleeding was successfully controlled.

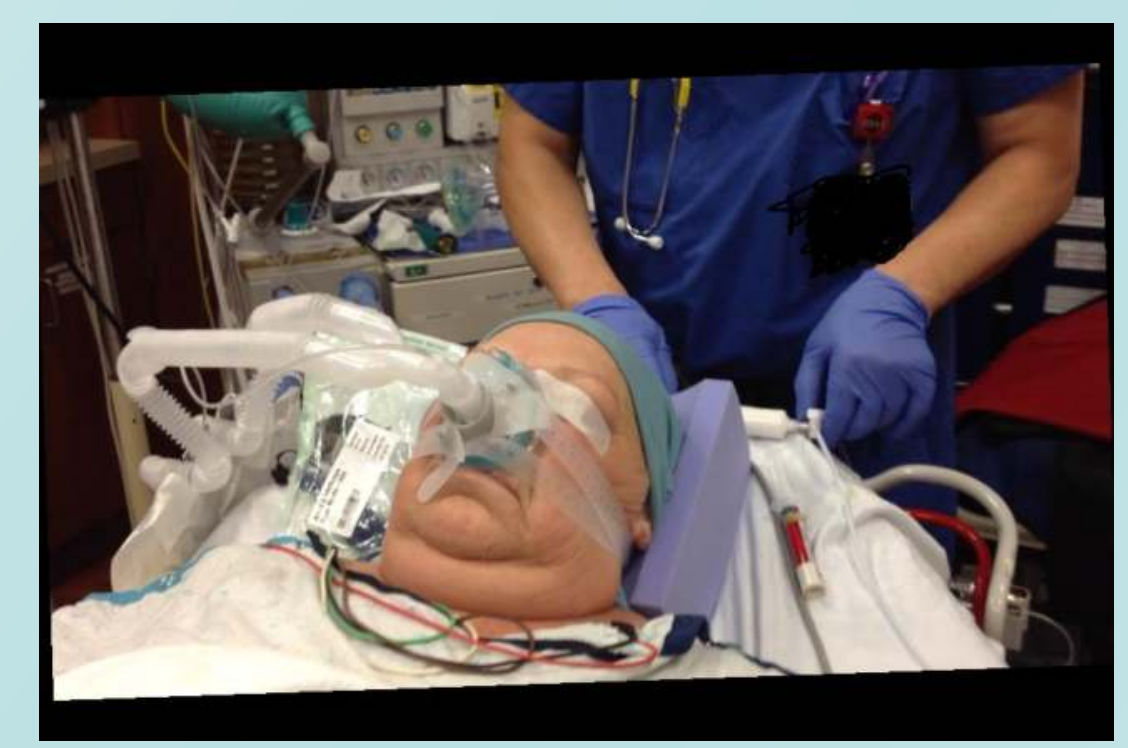

Photo 1. An infant mask was secured over the nose with head straps and connected to a breathing circuit and the anaesthesia machine.

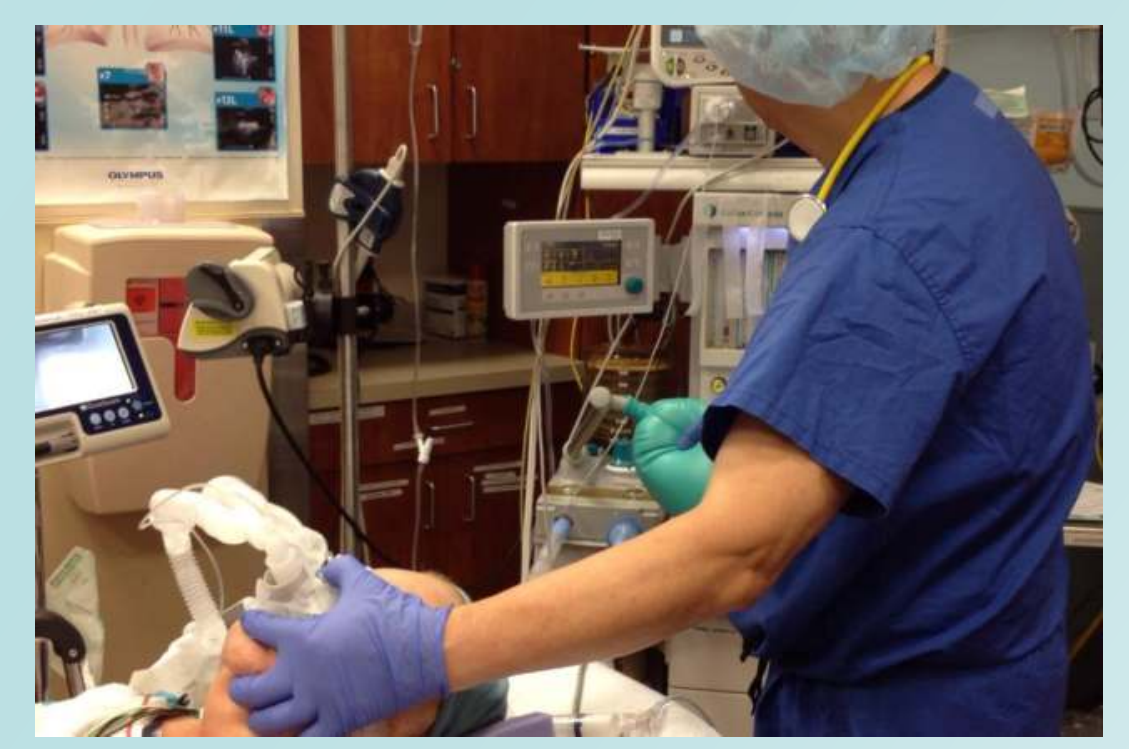

Photo 2. Nasal ventilation was performed by one anaesthesia provider after GA induction.

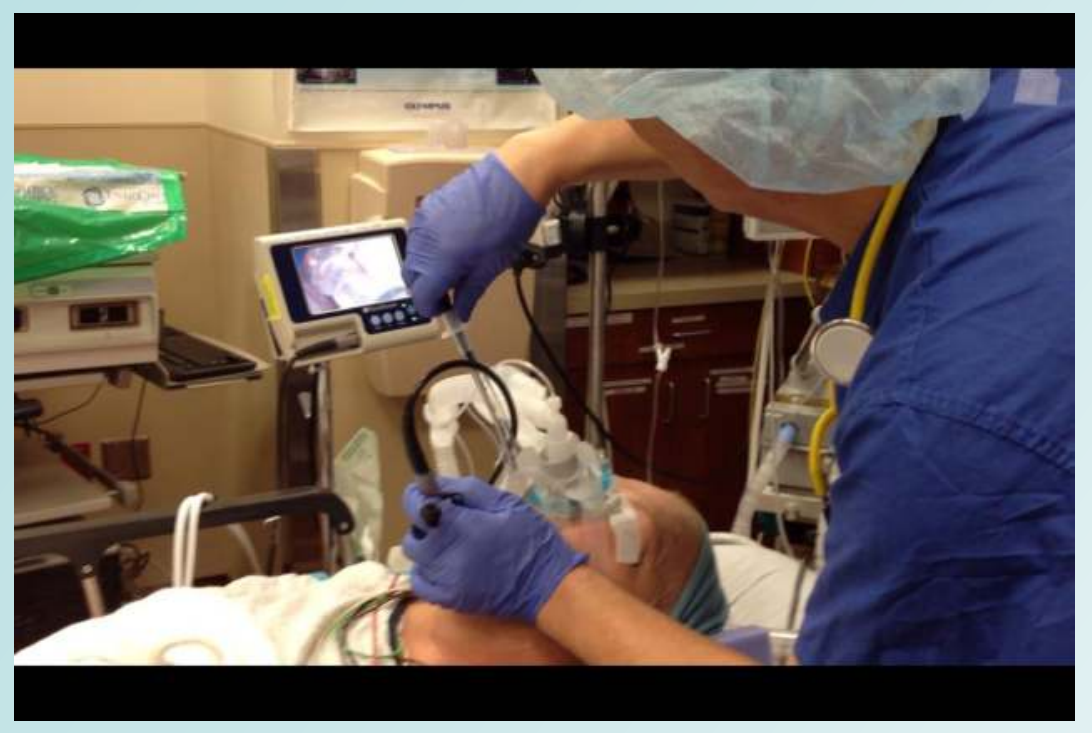

Photo 3. Apnoeic oxygenation was delivered with the nasal mask assembly during $\mathrm{VL}$ intubation.

Discussion: This nasal mask assembly provided continuous oxygenation in an obese patient with a difficult airway and poor face-mask fit during GA induction and VL ETI.

It prevented severe desaturation and may have improved patient safety.

Ref: 1. SAMBA $28^{\text {th }}$ AM, March 2013; 2.

www.TSEMask.com; 3. ASA AM (MC3009), Oct 2015

Learning Points: How to provide nasal CPAP preoxygenation in obese OSA patients, assisted nasal ventilation in anaesthetized patients with poor face-mask fit by one anaesthesia provider and continuous apnoeic oxygenation during VL endotracheal intubation. 